\title{
Razones para el uso de blogs por maestros en formación
}

\section{Jesús García Laborda ${ }^{\mathrm{a}}$}

${ }^{a}$ Universidad de Alcalá; Departamento de Filología Moderna; Calle Trinidad, 3, 28801 Alcalá de Henares (Madrid), email: jesus.garcialaborda@uah.es

\begin{abstract}
Although information technologies have been used in the university classroom since the early 1980s, their diffusion and depth is very different in Spain. In the careers of Humnidades and Social Science of the University of Alcalá they have been limited by resources certainly limited. Hence the need to obtain simple and accessible resources free of charge. Blogs are an excellent opportunity to develop the ability of future teachers to develop or combine their own creations and classroom tasks to give them maximum possible dissemination. This work succinctly presents the results of the reasons and use of blogs in teacher training. To do this, after the students of the Magisterium elaborated their blogs in groups, they were asked for their opinions regarding their use and functionality. Among the results, the combination of ludic and formative aspects stands out. This indicates that the use of blogs favors not only creativity, the ability to write, but is also highly motivating.
\end{abstract}

Keywords: blogs, teacher training, motivation, play elements, survey.

\section{Resumen}

Aunque las tecnologías de la información han sido utilizadas en el aula universitaria desde principios de los 80 , su difusión y profundidad es muy diferente en España. En las carreras de Humanidades y Ciencia Sociales de la Universidad de Alcalá se han visto limitadas por unos recursos ciertamente limitados. De ahí la necesidad de obtener recursos sencillos y accesibles gratuitamente. Los blogs constituyen una excelente oportunidad para desarrollar la capacidad de los futuros maestros de elaborar o combinar sus propias creaciones y tareas del aula para darles la máxima difusión posible. Este trabajo presenta sucintamente los resultados de las razones y uso de blogs en la formación de profesores. Para ello, tras elaborar los estudiantes de Magisterio sus blogs en grupos, se les solicitó sus opiniones respecto a su uso y funcionalidad de los mismos. Entre los resultados destaca la combinación de aspectos lúdicos y formativos. Esto indica que el uso de blogs favorece no sólo la creatividad, la capacidad de escritura sino que es, además, altamente motivador.

Palabras clave: blogs, formación del profesorado, motivación, elementos lúdicos, encuesta. 


\section{Introducción}

El uso de blogs se ha incrementado durante los últimos años debido al interés que suscitan tanto en profesores como en alumnos (MacDonald, 2015; Hughes, Liu \& Lim, 2016). Asimismo, los blogs permiten el uso de los mismos para desarrollar distintos estilos de competencia escrita y leída tanto de manera individual como colectiva (Morgan, 2015). Para muchos, los blogs, además, son la viva representación de una comunicación multimodal realista (Sandlin \& Peña, 2014).

Dentro de las competencias requeridas para los estudiantes en el siglo 21, los blogs permiten potenciar muchas de las áreas de desarrollo, especialmente el uso de la tecnología, conocimiento de lenguas extranjeras (figura 1) de manera interaccionista (Yang, 2018).

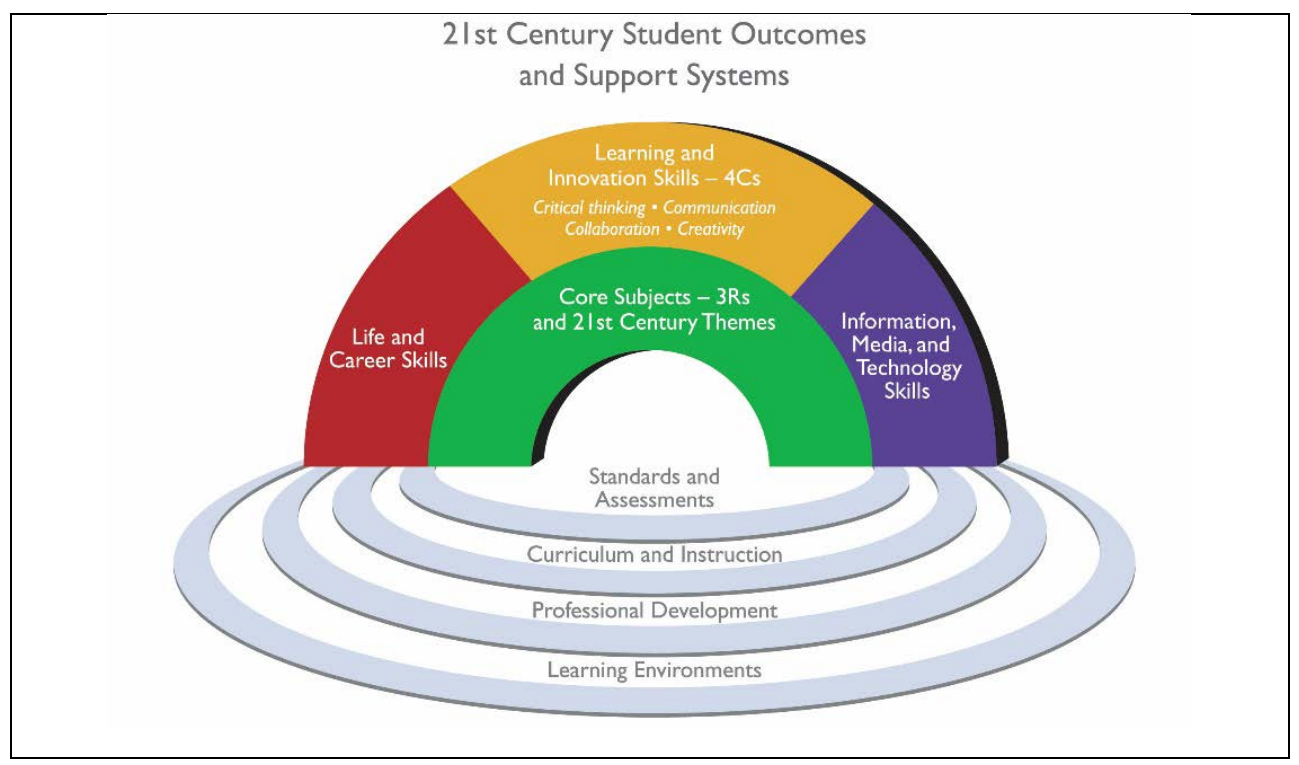

Fig. 1 Marco conceptual de las destrezas de aprendizaje del siglo 21 (fuente: www.P21.org)

\section{Objetivos}

El objetivo general del proyecto de innovación docente "Web 2.0 para maestros de inglés en formación (UAH/EV891)” era la incorporación de blogs como método de formación de profesores (Khan, 2017) para incorporar contenidos didácticos que sirviesen en su desarrollo profesional como docentes en la asignatura de Didáctica del inglés ( $4^{\circ}$ curso, Grado de Maestro de Primaria) con una participación de 23 alumnos (6 blogs en grupos de 3-4 alumnos). La creación de los blogs era una parte obligatoria evaluable (40\%) de la asignatura. Los objetivos de este proyecto son:

1) Familiarización a los alumnos con tecnología social;

2) Búsqueda de aplicaciones directas e indirectas de la tecnología para su docencia formal al graduarse;

3) Creación de objetos de aprendizaje; 
4) Adaptación de contenidos curriculares de Primaria para los trabajos en el propio aula; $\mathrm{y}$

5) La presentación como mini clases de cara a su práctica efectiva con audiencia en el aula.

Para conseguir estos objetivos deberían incluir una serie de módulos en el propio blog. García Laborda y Litzler (2017) describen un uso de blogs similar en la facultad de Ciencias Económicas de la Universidad de Alcalá. Según la propia instrucción del profesorado participante el blog debería incluir una serie de aplicaciones usadas específicamente con el fin de desarrollar su uso como parte de la enseñanza de lenguas. Los principios que regirían la selección de herramientas sería la que sugiere el American Council on the Teaching of Foreign Languages (ACTFL) para el uso de la tecnología según las habilidades lingüísticas a adquirir en el siglo XXI:

1) Comunicación: Ya que es importante que los niños del siglo 21 deben ser capaces de comunicar a través de redes sociales (Facebook, Storify, ejejot, Blogger, etc.);

2) Trabajo cooperativo: Puesto que deberán usar su lengua materna y las adquiridas adicionalmente para trabajar con otras personas de distinta lengua y cultura como miembros de equipos interdisciplinares usando Google sheets, diigo, PBWorks, etc.;

3) Pensamiento crítico: para ser capaces de conceptualizar, analizar y sintetizar así como negociar a través comunicaciones efectivas para resolver problemas y avanzar en sus relaciones personales y/o conocimiento (bubbl.us, lino-it, spycynodes);

4) Creatividad e innovación usando el lenguaje de manera original y creativa para realizar contribuciones adecuadas a través de Storyfy, PicMonkey, Big Huge labs...;

5) Alfabetización tecnológica, digital y de media usando paper.li, hootsuite, newsmap, animoto, netvibes o CC; $\mathrm{y}$

6) A éstos se añadirían otras facetas como flexibilidad y adaptabilidad, iniciativa y autodirectividad, habilidades sociales e interculturales y capacidad de responsabilidad y liderazgo.

\section{Desarrollo de la innovación}

Con el fin de que estos valores se practicasen y se pudiesen utilizar como una primera aproximación al uso de la tecnología para transmitir estos valores en primaria se pidió un blog que no sólo debería estar integrado por elementos electrónicos sino que tenía que ser acompañado por otras actividades no digitales como juegos, teatros de marionetas, etc. Para alcanzar este objetivo, el blog constaría de las siguientes partes:

1) Página web no dinámica con un tema de contenido y una explicación teórica;

2) Creación de dos videos para enseñar a alumnos de primaria materias relacionadas con el tema central;

3) Presentaciones realizadas en Power Point u otras aplicaciones de presentación;

(cc) EY-NC-ND 2018, Universitat Politècnica de València

Congreso In-Red (2018) 
4) Diseño de aplicaciones sociales para la promoción profesional (generalmente Linkedin); y

5) Enlaces a aplicaciones útiles para la enseñanza.

La figura 1 muestra la composición final de la interfaz principal de uno de estos blogs diseñados por los alumnos.

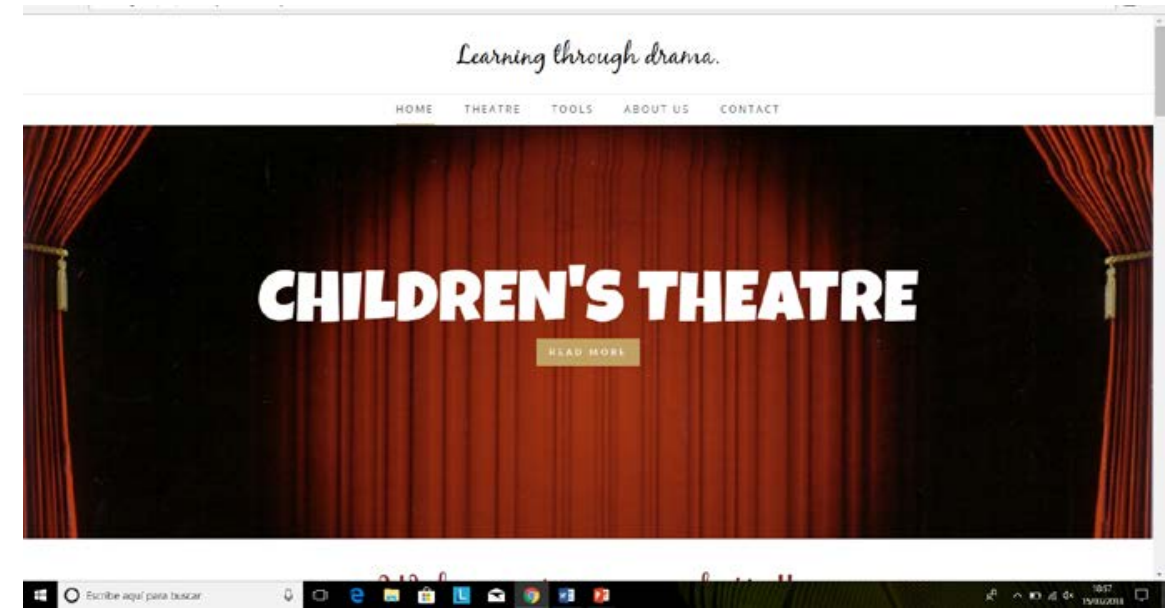

Fig. 1 Blog "Children's Theater" (https://funnytheatre.weebly.com/)

Este blog muestra de manera efectiva sus distintas partes. El objetivo último de la instrucción en educación sería la comunicación por tanto debemos considerar en nuestro constructo dicho objetivo último. En el diagrama dos se presenta el flujo direccional del constructo con el fin de llegar desde el input inicial hasta el output (figura 2).

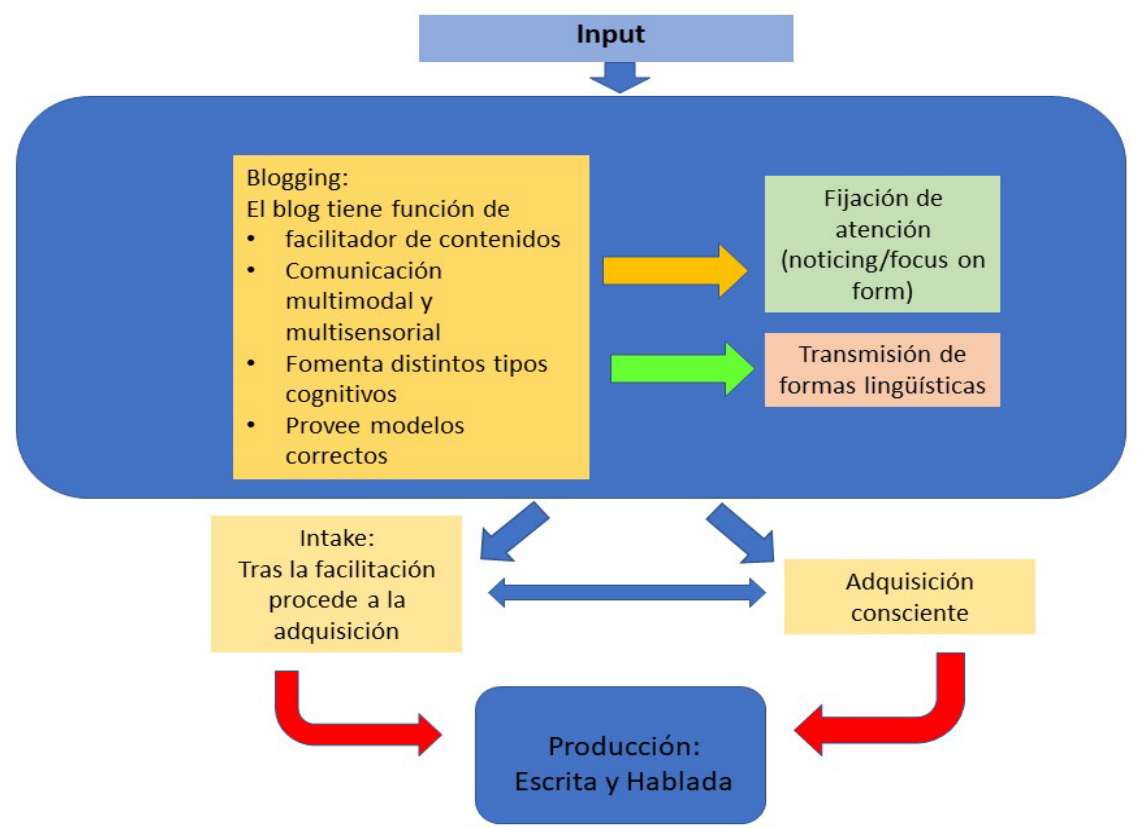

Fig. 2 Proceso de aprendizaje de la lengua a través de blogs (fuente propia)

(cc) EY-Nc-No 2018, Universitat Politècnica de València

Congreso IN-RED (2018) 
Como se ve en la figura 2, el blog sirve como medio de transmisión comunicativa no sólo de la lengua, sino que tiene una función de motivación. En el ejemplo señalado más arriba esta función la cumplen parcialmente los dos vídeos que, además, transmiten los contenidos lingüísticos de forma multimodal facilitando el aprendizaje de acuerdo a los distintos estilos cognitivos del alumno. Asimismo, puesto los estudiantes producen formas escritas, el alumno es capaz de crear y recibir modelos lingüísticos específicos especialmente porque los alumnos deben editar su producción, lo que, a su vez, requiere un proceso de monitorización y edición del propio trabajo que tiene un efecto en la fijación y en la atención a la forma y también un esfuerzo en la transmisión de las formas correctas. En nuestro ejemplo la variedad de estilos discursivos escritos se hace porque los futuros profesores crean lecturas que, a su vez, deben servir de lectura para potenciales alumnos (Yakut \& Aydin, 2017). Bien es cierto que, aunque en el blog sugerido el tema es muy elevado para los alumnos, en general, lo que se puede introducir una vez en el ejercicio de la enseñanza en educación primaria pueden ser cuentos con distintos estilos de letras (mayúscula, grande, pequeña, itálica, script) y colores que fomenten la creatividad de sus alumnos. Una vez los alumnos han percibido, entendido y fijados los contenidos se produce tanto una adquisición consciente como no consciente que, en última instancia, llevan al aprendizaje. Posteriormente los alumnos podrán producir ejercicios escritos para sus compañeros (y en el futuro para sus alumnos potenciales).

\section{Resultados}

En primera instancia el producto del blog mismo es un resultado ya que los alumnos han desarrollado un objeto (o varios) de aprendizaje que es transferible a su realidad profesional y, por consiguiente, debe ser valorado por sí mismo. Los alumnos, también, reconocen que tiene otros efectos que se reflejan en la figura 3. Para ello realizaron una encuesta libre a través de Survey Monkey que respondieron demanera voluntaria y que trataba de encontrar beneficios y problemas de la implementación del proyecto. 


\section{¿Cuál cree que podría ser el beneficio si se usasen blogs creados por los alumnos en enseñanza primaria?}

Respondidas: 15 Omitidas: 0

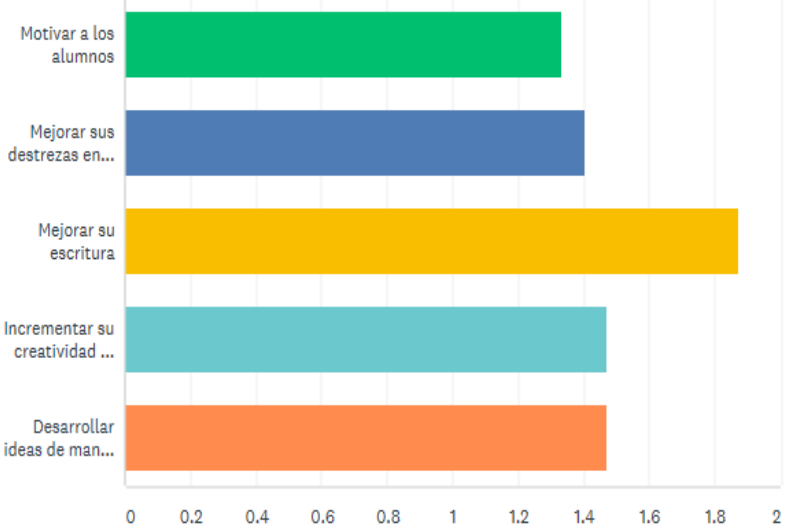

Fig. 3 Proceso de aprendizaje de la lengua a través de blogs

La figura 3 muestra que los alumnos encuentran una transferencia directa del trabajo realizado que además tiene un efecto significativo en la motivación, en la mejora de destrezas lingüísticas y, por supuesto, muy especialmente en la escritura (también Akdag \& Özcan, 2017). Así los alumnos muestran sus opiniones valorando con 2 su afirmación absoluta y un 0 su negativa absoluta. Así pues, su máxima valoración se va a la mejora de la escritura. Asimismo, sirve para fomentar la creatividad en el aula (azul claro) y el desarrollo de ideas y la mejora de destrezas escritas (azul oscuro).

\section{Conclusiones}

La creación de blogs representa una inmensa cantidad de beneficios en la formación de futuros maestros que se forman en el desarrollo de competencias exigibles y necesarias en el siglo XXI. Como se ha visto es un excelente medio para aprendizaje de lenguas extranjeras tanto para los creadores como para los receptores potenciales.

Este trabajo ha mostrado el proceso de aprendizaje y desarrollo lingüístico ejemplificado en un blog desarrollado para la asignatura de Didáctica de la Lengua Inglesa correspondiente al Grado de Maestro de Educación primaria de la Universidad de Alcalá. Obviamente, las propias limitaciones del estudio estriban en su eminente practicidad contrastada con otros trabajos en el ámbito del uso de blogs por maestros y, por supuesto, en la propia limitación de la aplicación ya que las clases en la escuela de Magisterio de la UAH nunca tienen más de 25 alumnos y ésta específicamente tan solo 20.

Por tanto, futuros trabajos deberían basarse en una muestra mucho más amplia de alumnos y en el análisis de sus propias dificultades. También se podría estudiar el efecto de la 
creación de esos blogs en los propios estudiantes y en su uso fuera y dentro de clase. En resumen, ésta es una primera aproximación que tiene una diferencia en la aplicabilidad inmediata del objeto desarrollado y en la propia percepción de su utilidad por los propios maestros en formación.

\section{Referencias}

AKDAG, E., \& ÖZKAN, Y. (2017). Enhancing writing skills of EFL learners through blogging. Reading Matrix: An International Online Journal, 17(2), 79-95.

García LABORDA, J., \& LitZler, M. F. (2017). English for business: Student responses to language learning through social networking tools. EST Today: Journal of English for Specific Purposes at Tertiary Level, 5(1), 91-107.

HughES, J. E., LiU, S., \& LiM, M. (2016). Technological modeling: Faculty use of technologies in preservice teacher education from 2004 to 2012. Contemporary Issues in Technology and Teacher Education (CITE Journal), 16(2) Retrieved from https://search-proquestcom.ezproxy.uned.es/docview/1871576920?accountid=14609

KHAN, A. (2017). Blog-based professional development of English teachers in Mumbai: The potential of innovative practice under scrutiny.Australasian Journal of Educational Technology, 33(4), 88-106.

MACDONALD, D. (2015). Teacher-as-knowledge-broker in a futures-oriented health and physical education. Sport, Education and Society, 20(1), 27-41.

Morgan, H. (2015). Creating a class blog: A strategy that can promote collaboration, motivation, and improvement in literacy. Reading Improvement, 52(1), 27-31.

SANDLIN, J. K., \& PEÑA, E. V. (2014). Building authenticity in social media tools to recruit postsecondary students. Innovative Higher Education, 39(4), 333-346.

SEJDIU, S. (2017). Are listening skills best enhanced through the use of multimedia technology. Digital Education Review, (32), 60-72.

YAKUT, A. D., \& AYdin, S. (2017). An experimental study on the effects of the use of blogs on EFL reading comprehension. Innovation in Language Learning and Teaching, 11(1), 1-16.

YANG, S. J. (2018). Language learners' perceptions of having two interactional contexts in eTandem. Language Learning \& Technology, 22(1), 42-51. 